\title{
Multi-model ensemble hydrologic prediction and uncertainties analysis
}

\author{
SHANHU JIANG, LILIANG REN, XIAOLI YANG, MINGWEI MA \& YI LIU \\ State Key Laboratory of Hydrology-Water Resources and Hydraulic Engineering, Hohai University, Nanjing 210098, \\ China \\ rll@,hhu.edu.cn
}

\begin{abstract}
Modelling uncertainties (i.e. input errors, parameter uncertainties and model structural errors) inevitably exist in hydrological prediction. A lot of recent attention has focused on these, of which input error modelling, parameter optimization and multi-model ensemble strategies are the three most popular methods to demonstrate the impacts of modelling uncertainties. In this paper the Xinanjiang model, the Hybrid rainfallrunoff model and the HYMOD model were applied to the Mishui Basin, south China, for daily streamflow ensemble simulation and uncertainty analysis. The three models were first calibrated by two parameter optimization algorithms, namely, the Shuffled Complex Evolution method (SCE-UA) and the Shuffled Complex Evolution Metropolis method (SCEM-UA); next, the input uncertainty was accounted for by introducing a normally-distributed error multiplier; then, the simulation sets calculated from the three models were combined by Bayesian model averaging (BMA). The results show that both these parameter optimization algorithms generate good streamflow simulations; specifically the SCEM-UA can imply parameter uncertainty and give the posterior distribution of the parameters. Considering the precipitation input uncertainty, the streamflow simulation precision does not improve very much. While the BMA combination not only improves the streamflow prediction precision, it also gives quantitative uncertainty bounds for the simulation sets. The SCEM-UA calculated prediction interval is better than the SCE-UA calculated one. These results suggest that considering the model parameters' uncertainties and doing multi-model ensemble simulations are very practical for streamflow prediction and flood forecasting, from which more precision prediction and more reliable uncertainty bounds can be generated.
\end{abstract}

Key words input error modelling; parameter optimization; Bayesian model averaging; uncertainty analysis

\section{INTRODUCTION}

A hydrological model is an approximate description of the complicated hydrologic phenomena that occur in nature. It is an effective method for understanding the complex hydrologic cycle process, and is also a powerful tool for solving the practical hydrological problems. Since the $1850 \mathrm{~s}$, hydrological models have developed from empirical models to conceptual models and thus to distributed models. Although the precision of hydrological prediction has increased with the development of models, in practice there are still inevitably different modelling uncertainties, i.e. input errors, parameter uncertainties and model structural errors (Beven 2000). Numerous studies have focused on hydrological modelling uncertainties analysis (Ajami et al. 2007, Duan et al. 2007), and highlighted that input error modelling, parameter optimization and multi-model ensemble strategies are the three most popular methods to demonstrate the impacts of hydrological prediction uncertainties. Ajami et al. (2007) introduced a normal distributed error multiplier to reduce the precipitation input uncertainty. For the parameter uncertainty, there are two different estimation methods: the first assumes that for a certain basin and model, only one optimal parameter set can be found, and the typical algorithms are GA and SCE-UA; the other assumes that for a given basin and model, the model parameters are one set of random variables which obey a certain joint probability distribution and the typical algorithms are GLUE and SCEM-UA. There are numerous available models, and each has a different focus on describing the hydrological physical processes. No single model can completely describe the principles of watershed rainfall-runoff in all conditions. So ensemble approaches based on several models can help reduce the model structure uncertainty and improve the hydrological prediction precision.

This study focuses on applying a multi-model approach to perform ensemble streamflow simulation and uncertainties analysis. The primary innovation of this paper is that we compared the affects of two different parameters optimization methods, i.e. SCE-UA and SCEM-UA, on hydrological prediction uncertainty. 


\section{STUDY AREA AND DATA}

Mishui basin, a tributary of Xiangjiang River with a drainage area of $9972 \mathrm{~km}^{2}$ above the Ganxi hydrologic station, was selected as the study area (Fig. 1). The basin is located southeast of Hunan Province in south China and extends from longitudes $112.85^{\circ} \mathrm{E}$ to $114.20^{\circ} \mathrm{E}$ and latitudes $26.00^{\circ} \mathrm{N}$ to $27.20^{\circ} \mathrm{N}$. The basin has a complex topography, with elevations ranging from 49 to $2093 \mathrm{~m}$ a.s.l. The climate is a humid subtropical monsoon type, with an average temperature of approximately $18.0^{\circ} \mathrm{C}$ and mean annual precipitation of approximately $1561 \mathrm{~mm}$.

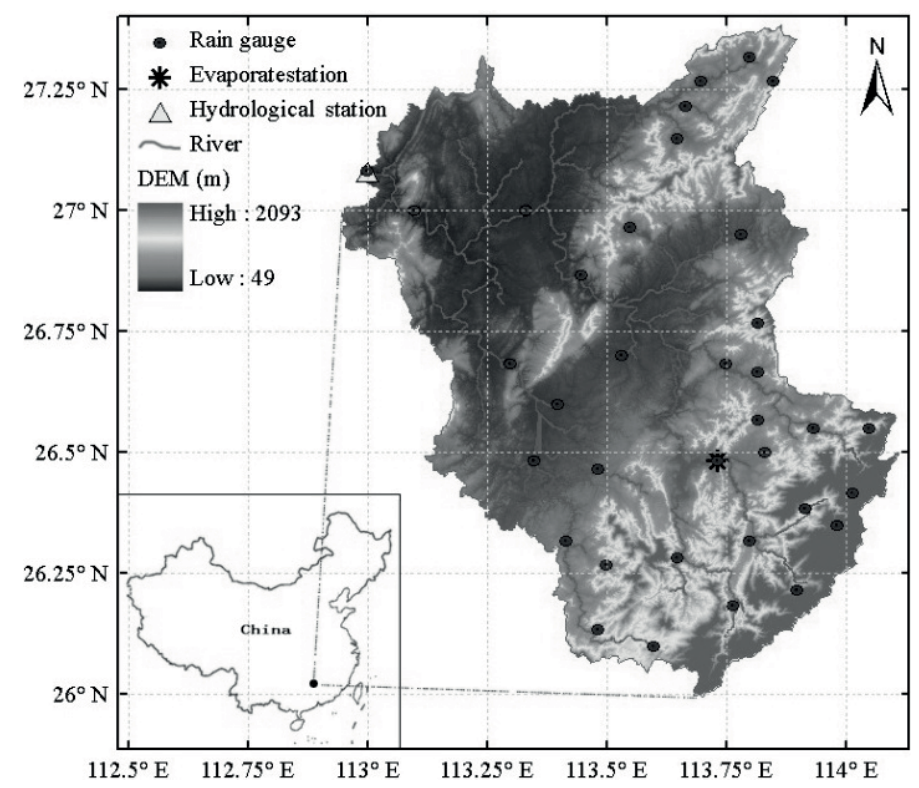

Fig. 1 Map of Mishui basin in south China.

Observed daily precipitation (P) data for 2003 to 2008 were derived from 35 raingauge stations. For the same period, daily streamflow and potential evapotranspiration (PET) data were collected from the Ganxi hydrologic station and Wulipai evaporation station, respectively. The inverse distance weighting of the three nearest raingauges was used to obtain the spatially distributed precipitation database of Mishui basin.

\section{METHODOLOGY}

\section{Hydrological models}

The Xinanjiang model (XAJ) is a well-known conceptual hydrological model developed by Zhao in the 1970s (Zhao 1992). The XAJ model has been successfully and widely used in the humid and semi-humid regions of China. Here, a sub-basin-structured XAJ model for streamflow simulation was constructed. The simulation was performed by computing the runoff and dividing the runoff types within each sub-basin. The slope and river network convergence processes were then integrated to obtain the streamflow series of the hydrologic station. The Hybrid rainfall-runoff model (HYB) is a modified version of the XAJ model (Hu et al. 2005). Numerous field studies show that runoff within a basin is mainly generated by two mechanisms: infiltration excess (Horton) runoff and saturation excess (Dunne) runoff. The HYB model combines the two runoff mechanisms by means of combining the spatial distribution curves of soil tension water storage capacity and infiltration capacity. For a detailed description of the mechanisms and application of the HYB model used here see Hu et al. (2005). HYMOD is simple conceptual lumped hydrological model developed by Moore in the 1980s (Moore 1985; hereinafter referred to as HYM). The HYM consists of a simple rainfall excess model connected to two series of linear reservoirs to route surface and subsurface flow. Here, we added an evaporation reduction factor $K$ and a river network routing Muskingum-Cunge model to the original HYM. Table 1 shows the main characteristics of the three models. 
Table 1 The main characteristics of the three hydrological models.

\begin{tabular}{llllll}
\hline Model & Input & Parameter & Runoff generation & Evaporation calculation & Runoff division \\
\hline XAJ & P, PET & 15 & Saturation excess & Three layers & Surface, subsurface, groundwater \\
HYB & P, PET & 14 & Infiltration and saturation excess & Three layers & Surface, groundwater \\
HYM & P, PET & 9 & Saturation excess & One layer & Surface, groundwater \\
\hline
\end{tabular}

The models were operated daily for 15 sub-basins in Mishui basin. The period January 2003 to December 2005 was selected as the calibration period, and January 2006 to December 2008 was selected as the validation period.

\section{Input error modelling}

The main inputs of the hydrological model are the hydrometeorological data sets, of which precipitation is the most important. In this study, we adopted an error multiplier to consider the precipitation input uncertainty:

$$
\begin{aligned}
& P_{t}=\varphi_{t} \cdot \stackrel{\square}{P_{t}} \\
& \phi_{t}=N\left(m, \sigma_{m}^{2}\right)
\end{aligned}
$$

where $P_{t}$ is the measured precipitation at time step $t ; P_{t}$ is the modified precipitation; and $\varphi_{t}$ is a normal error multiplier with a mean value of $m$ and a variance of $\sigma_{m}^{2}$ at time step $t$, and $t$ is the total number of simulation days. In this study, we assume that $m \in[0.9,1.1]$ and $\sigma_{m}^{2} \in\left[10^{-5}, 10^{-3}\right]$.

\section{Parameter optimization}

The shuffled complex evolution (SCE-UA) is an effective and efficient global optimization algorithm proposed by Duan et al. (1992) and has been widely used in hydrological model parameter optimization. The SCE-UA combines the direction-searching of deterministic, non-numerical methods and the robustness of stochastic, non-numerical methods. It adopts competition evolution theory, concepts of controlled random search, the complex shuffling method and downhill simplex procedures to obtain a global optimal estimation. The detailed calculation steps of SCE-UA can be found in Duan et al. (1992). The SCEM-UA was built upon the principles of SCE-UA. Vrugt et al. (2003) combined the strengths of the Monte Carlo Markov Chain sampler with the concept of complex shuffling from SCE-UA to form this algorithm that not only provides the most probable parameter set, but also estimates the uncertainty associated with estimated parameters. The SCEMUA can simultaneously identify the most likely parameter set and its associated posterior probability distribution in every model run (Ajami et al. 2007). The detailed calculation steps of SCEM-UA can be found in Vrugt et al. (2003). Here, the initial samples and the computation times were set at 5000 and 10000 , respectively.

\section{Bayesian model averaging}

BMA is a probabilistic scheme for model combination that derives the consensus prediction from competing predictions using likelihood measures as model weights. BMA has previously been primarily used to generalize linear regression applications. Duan et al. (2007) successfully used the BMA to combine multi-model for hydrologic ensemble prediction, which can develop more skilful and reliable probabilistic prediction. The advantage of the BMA is that the weights are directly bound with individual model simulation, i.e. a better performing model can receive a higher weight than a poorly performing one. A more robust and stable result can be obtained when the BMA method is used to combine different simulations. In this study, we use the BMA to merge the streamflow simulations from the three different hydrological models. The detailed calculation steps of the BMA method can be found in Duan et al. (2007). 


\section{Evaluation statistics}

The statistical validation indices, the Nash-Sutcliffe coefficient (NSCE) and relative bias (BIAS), were employed to evaluate hydrologic model performance based on the observed and simulated streamflow series (Jiang et al. 2012). The statistical validation indices of the containing ratio (CR), average bandwidth (B), and average deviation amplitude (D) were adopted to evaluate the prediction bounds of the hydrological models (Xiong et al. 2009). CR denotes the ratio of the number of observed streamflows enveloped by prediction bounds to the total number of observed hydrographs, expressed as a percentage. B represents the average bandwidth of the whole prediction bounds. D denotes the actual discrepancy between the trajectories consisting of the middle points of the prediction bounds and the observed hydrograph, and shows the symmetry with respect to the observed discharges and the middle point of the prediction bounds.

\section{RESULTS AND DISCUSSION}

To consider the three sources of hydrological prediction uncertainties, we adopt three cases to perform the streamflow simulation. Case 1 just considers the model parameter uncertainty using the SCE-UA and SCEM-UA algorithms. Case 2 introduces a normal distributed error multiplier and combines the parameter optimization algorithms to consider the model input and model parameters' uncertainties. Case 3 combines the simulation sets calculated from the three models in Case 2 by using BMA to comprehensively consider the model input, model parameter and model structure uncertainties. Figure 2 shows the performances of the streamflow simulation based on the SCE-UA and SCEM-UA algorithms and the three simulation cases. Figure 3 shows the BMA combined streamflow series and the $95 \%$ confidence interval of Case 3 . All three models have good hydrologic prediction applicability in Mishui basin: the XAJ model is the optimal one, then the HYB model, and the HYM model least good. Both the two parameter optimization algorithms can generate good streamflow simulations. The SCEM-UA can imply parameter uncertainty and give the posterior distribution of the parameters, and also, using the 15000 sets simulation, can calculate the prediction uncertainty boundary. Considering the precipitation input uncertainty does not improve the precision of the three models simulated streamflows, this may be due to: (1) in Mishui basin, there are dense raingauge data and the precipitation input uncertainty is relatively small; (2) in the model parameter, we set an evaporation reduction factor parameter $K$ and this parameter can imply some input uncertainty. For the SCE-UA and SCEM-UA algorithms, the BMA combinations of the

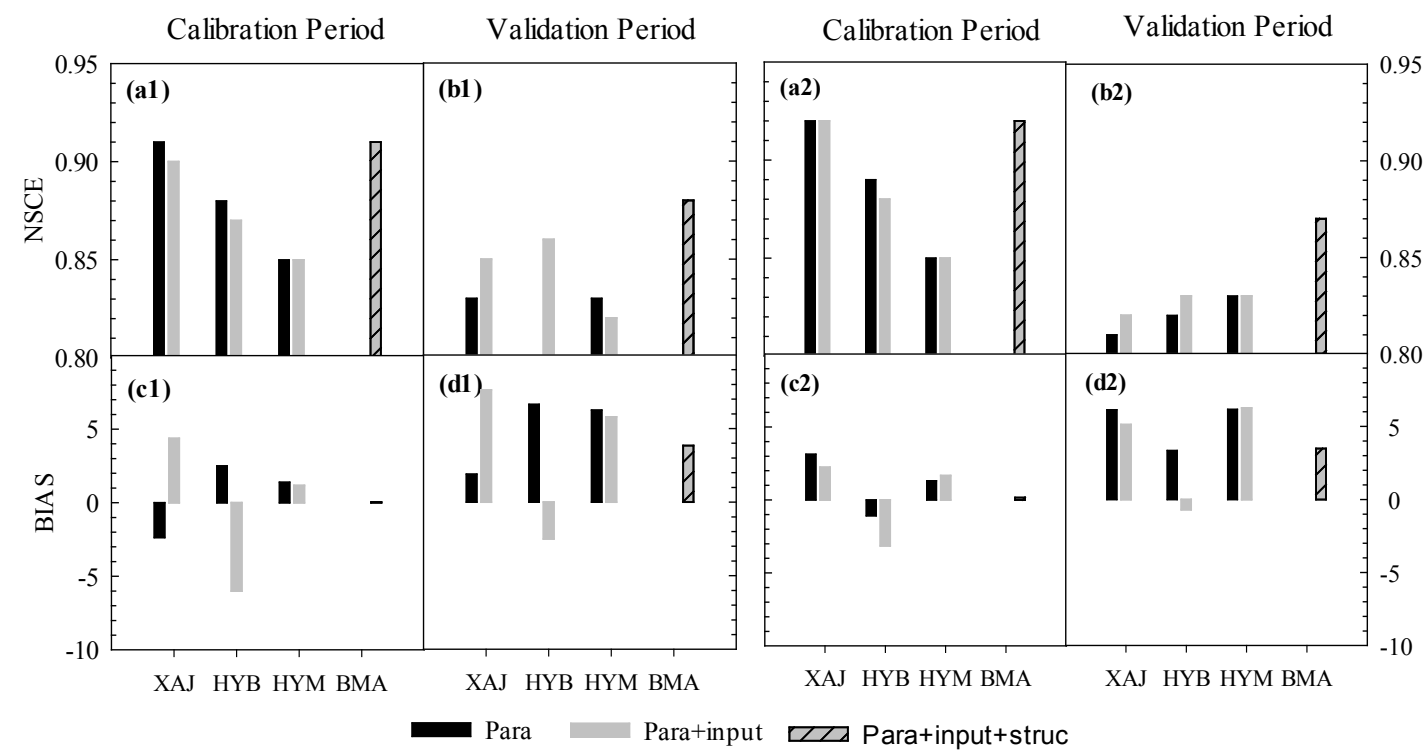

Fig. 2 The precision performances of the SCE-UA (left) and SCEM-UA (right) based streamflow simulation at different simulation cases. 


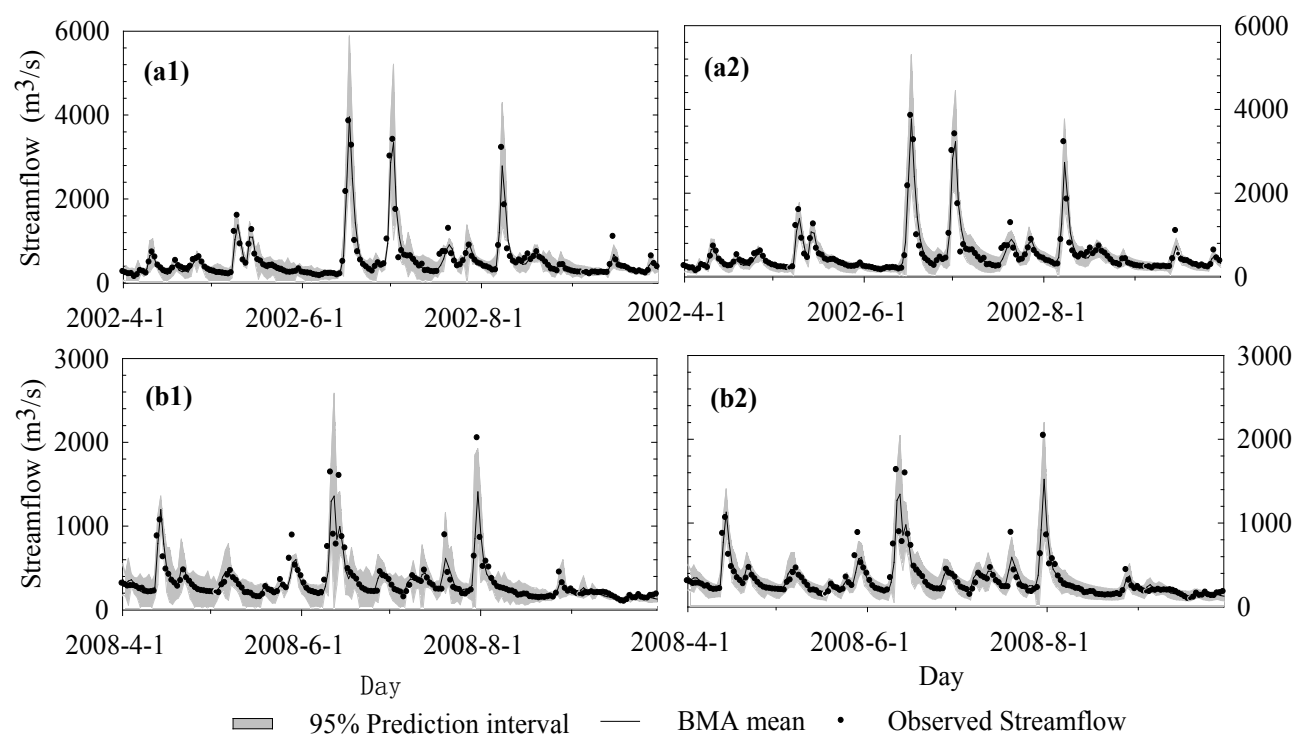

Fig. 3 The BMA combined streamflow series and the 95\% confidence interval from the SCE-UA (left) and SCEM-UA (right) based simulations. Note: For the SCE-UA, we use a Markov chain Monte Carlo sampling method (sampling 1000 times) to calculate the prediction interval (Duan et al., 2007).

Table 2 The reliability performance of the calculated 95\% confidence interval at different simulation cases.

\begin{tabular}{|c|c|c|c|c|c|c|c|}
\hline \multicolumn{2}{|c|}{ Cases } & \multicolumn{3}{|c|}{ SCE-UA (Sampling 1000 times) } & \multicolumn{3}{|c|}{ SCEM-UA } \\
\hline & & $\mathrm{CR}(\%)$ & $\mathrm{B}\left(\mathrm{m}^{3} / \mathrm{s}\right)$ & $\mathrm{D}\left(\mathrm{m}^{3} / \mathrm{s}\right)$ & $\mathrm{CR}(\%)$ & $\mathrm{B}\left(\mathrm{m}^{3} / \mathrm{s}\right)$ & $\mathrm{D}\left(\mathrm{m}^{3} / \mathrm{s}\right)$ \\
\hline \multirow[t]{7}{*}{$\mathrm{CP}$} & XAJ (Para) & 59.31 & 152.87 & 58.20 & 78.65 & 169.17 & 52.31 \\
\hline & XAJ (Para+input) & 74.86 & 200.15 & 60.78 & 79.06 & 169.78 & 51.79 \\
\hline & HYB (Para) & 75.05 & 258.66 & 74.08 & 80.34 & 222.25 & 64.41 \\
\hline & HYB (Para+input) & 81.07 & 273.01 & 65.39 & 78.97 & 225.23 & 67.60 \\
\hline & HYM (Para) & 71.40 & 225.70 & 63.08 & 85.26 & 237.97 & 62.91 \\
\hline & HYM (Para+input) & 68.57 & 212.49 & 63.29 & 87.68 & 254.06 & 64.01 \\
\hline & BMA (Para+input + struc) & 90.19 & 315.60 & 56.70 & 95.62 & 271.15 & 55.03 \\
\hline \multirow[t]{7}{*}{ VP } & XAJ (Para) & 62.32 & 183.64 & 71.21 & 80.47 & 188.68 & 64.41 \\
\hline & XAJ (Para+input) & 73.81 & 220.50 & 74.63 & 81.48 & 190.31 & 63.07 \\
\hline & HYB (Para) & 71.99 & 289.95 & 82.84 & 80.66 & 244.40 & 74.13 \\
\hline & HYB (Para+input) & 82.66 & 285.44 & 71.71 & 80.38 & 249.24 & 77.14 \\
\hline & HYM (Para) & 68.61 & 270.26 & 77.88 & 86.77 & 261.23 & 76.62 \\
\hline & HYM (Para+input) & 69.16 & 252.23 & 76.84 & 88.96 & 278.24 & 77.31 \\
\hline & BMA (Para+input+struc) & 90.97 & 348.56 & 69.74 & 95.17 & 303.04 & 66.06 \\
\hline
\end{tabular}

Notes: in the table, Para indicates considering the model parameter uncertainty, Para+input means considering the model input and model parameter uncertainties, Para + input + struc revels considering the model input, model parameter and model structure uncertainties.

simulation sets calculated from Case 2 improve the streamflow prediction precision in terms of the highest NSCE and the smallest BIAS, especially in the validation period. Figures 2 and 3 show that the BMA combining multi-models for the ensemble streamflow simulation can effectively improve the prediction precision.

Table 2 shows the reliability performance of the calculated $95 \%$ confidence interval at different simulation cases. For both the SCE-UA and SCEM-UA algorithms, the reliability performance of the $95 \%$ confidence interval calculated from the BMA combined streamflows is much better than the results calculated from each signal model simulated streamflows. The BMA combination calculated 95\% confidence interval has higher CR and better D, while it also has a larger B. The table also demonstrates that the reliability performance of the SCEM-UA based predict interval in terms of CR and D is generally superior to that of the SCE-UA based predict interval, which indicates that the SCEM-UA algorithm has advantage in prediction uncertainty boundary estimation than the SCE-UA algorithm (Fig. 3). Overall of the whole table, the BMA combining multi-model 
to do the ensemble hydrologic simulation can effectively calculate much more reliable uncertainty bounds.

\section{CONCLUSIONS AND SUGGESTIONS}

The XAJ model, HYB model and HYM model all have good applicability in Mishui basin. Both the SCE-UA and SCEM-UA parameter optimization algorithms can generate good streamflow simulations; in particular, the SCEM-UA can imply parameter uncertainty and give the posterior distribution of the parameters. The precipitation input uncertainty consideration does not improve the streamflow simulation precision. The BMA combination of the simulation sets calculated from a single model improves the streamflow prediction precision, and quantifies the uncertainty bounds for the simulation sets. The calculated $95 \%$ prediction interval from the merged simulations of SCEM-UA is much better than that calculated from the SCE-UA based simulations. These results suggest that considering the model parameters uncertainties and doing multi-model ensemble simulations are very practical for streamflow prediction and flood forecasting, from which we can generate more precision prediction and more reliable uncertainty bounds. However, in practical applications this will inevitably increase the amount of computation. Here, the spatial resolution of the three sub-basins based hydrological models is low. The computational efficiency is high and can be well applied in ensemble hydrological prediction. The development direction of hydrological models is towards distributed models with high spatial and temporal resolution, describing the temporal and spatial heterogeneity of hydrological process in more detail. But such a distributed model will require large computation resources and calculation time. Both types of models have advantages and disadvantages. Thus, in future, we can combine the high-resolution "single" model simulation scheme and the low-resolution multi-model "ensemble" simulation method to improve the hydrological prediction precision and further understand the spatial and temporal variation of the hydrological process.

Acknowledgements The current study was supported by the Programme of Introducing Talents of Discipline to Universities by the Ministry of Education and the State Administration of Foreign Experts Affairs, China (the 111 Project, No. B08048), the National Natural Science Foundation of China (Grant No. 41323001), and the Special Basic Research Fund by the Ministry of Science and Technology, China (No. 2011IM011000).

\section{REFERENCES}

Ajami, N. K., Duan, Q. Y. and Sorooshian S. (2007) An integrated hydrologic Bayesian multi-model combination framework: Confronting input, parameter, and model structural uncertainty in hydrologic prediction. Water Resources Research 43, W01403.

Beven, K. (2000) Rainfall-Runoff Modelling. Chichester: Wiley.

Duan, Q. Y., Sorooshian, S. and Gupta, V. (1992) Effective and efficient global optimization for conceptual rainfall-runoff models. Water Resources Research 28(4), 1015-1031.

Duan, Q. Y., et al. (2007) Multi-model ensemble hydrologic prediction using Bayesian model averaging. Advances in Water Resources 30, 1371-1386.

$\mathrm{Hu}, \mathrm{C} . \mathrm{H}$. , et al. (2005) A modified Xinanjiang model and its application in Northern China. Nordic Hydrology 36(2), $175-192$.

Jiang S. H., et al. (2012) Comprehensive evaluation of multi-satellite precipitation products with a dense rain gauge network and optimally merging their simulated hydrological flows using the Bayesian model averaging method. Journal of Hydrology 452-453, 213-225.

Moore, R. J. (1985). The probability-distributed principle and runoff production at point and basin scales. Hydrological Sciences Journal 30(2), 273-297.

Vrugt, J. A. et al. (2003) A Shuffled Complex Evolution Metropolis algorithm for optimization and uncertainty assessment of hydrologic model parameters. Water Resources Research 39, 1201.

Xiong, L. H., et al. (2009) Indices for assessing the prediction bounds of hydrological models and application by generalized likelihood uncertainty estimation. Hydrological Science Journal 54(5), 852-871.

Zhao, R. J. (1992) The Xinanjiang model applied in China. Journal of Hydrology 135(1-4), 371-381. 